\title{
Imprint cytology improves accuracy of computed tomography-guided percutaneous transthoracic needle biopsy
}

\author{
Y-C. Chang*, C-J. Yu , W-J. Lee*, S-H. Kuo`, C-H. Hsiao+, I-S. Jan`, F-C. Hu ${ }^{\S}$, \\ H-M. Liu*, W-K. Chan ${ }^{\dagger}$ and P.C. Yang ${ }^{\#}$
}

ABSTRACT: The aim of the present study was to investigate whether imprint cytology can improve the diagnostic accuracy of computed tomography-guided transthoracic core biopsy.

Between October 1997 and June 2004, thoracic lesions in 622 patients underwent biopsy using 19-gauge coaxial guiding needles and 20-gauge biopsy needles under computed tomography guidance. Touch imprint cytology and histopathology were performed for all biopsy specimens.

Of these lesions, 431 (74.1\%) were diagnosed as malignant, 151 (25.9\%) as benign and 40 (6\%) as nondiagnostic. Imprint cytology plus histology shows an improved diagnostic accuracy of $\mathbf{9 6 . 4 \%}$ compared with that of imprint cytology alone $(\mathbf{9 2 . 3 \% )}$ or histopathology alone $(\mathbf{9 3 . 0} \%)$. Procedure-related complications requiring further treatment occurred in eight (1.4\%) patients.

In conclusion, imprint cytology combined with histopathology can improve the diagnostic accuracy of computed tomography-guided transthoracic needle biopsy.

\section{KEYWORDS: Computed tomography, cytology, diagnostic accuracy, needle lung biopsy}

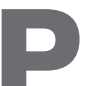

ercutaneous transthoracic needle biopsy (TNB) is an important diagnostic tool in the management of lung and mediastinal lesions [1-5]. Fine-needle aspiration (FNA) with computed tomography (CT) guidance has an accuracy and sensitivity of $76-95 \%$ for the detection of malignancy in solitary lung nodules [3-5]. Automated biopsy needles can acquire more core specimens and increase the diagnostic sensitivity to $84-96 \%[6,7]$. Coaxially guided needle biopsy minimises the risk associated with repeated pleural penetration and increases the volume of tissue retrieved compared with aspiration cytology or single-shot core biopsy. The diagnostic sensitivity for malignancy is $77-96 \%$ and the specificity for benign disease is $91-94 \%$ with automated coaxial core biopsy $[1,2,8]$.

Efforts to increase the diagnostic accuracy of image-guided TNB include frozen-section pathology [9] or FNA cytology combined with core biopsy under CT fluoroscopic guidance [10]. Touch imprint cytology is useful for diagnosing metastasis in surgically removed lymph nodes in breast cancer and better than conventional haematoxylin-eosin staining of paraffin sections $[11,12]$. However, data regarding imprint cytology and TNB are very limited. Recently, PAULOSE et al. [13] showed that imprint cytology could assist rapid diagnosis of lung cancer metastasis in mediastinal lymph nodes following CT-guided TNB. LIAO et al. [14] demonstrated improved diagnostic accuracy by using imprint cytology following ultrasound (US)-guided TNB of peripheral lung lesions. The role of touch imprint cytology in CT-guided coaxial core biopsy of intrathoracic lesions has not been investigated. The objective of the present study was to evaluate whether touch imprint cytology as an adjunct to CT-guided coaxial core biopsy can improve diagnostic accuracy for thoracic lesions.

\section{METHODS AND MATERIALS}

\section{Study subjects}

Tissue specimens from 622 patients who underwent CT-guided TNB of thoracic lesions between October 1997 and June 2004 at the National Taiwan University Hospital (National Taiwan University, Taipei, Taiwan) were examined using both histopathology and touch imprint cytology. Clinicians at the National Taiwan University Hospital prefer to assign patients with a peripheral lung lesion or pleural lesion to undergo USguided TNB as this is a quicker and less expensive method [15]. Patients were referred for CT-guided TNB if US-guided biopsy studies were considered unfruitful.

All patients were requested to discontinue any anticoagulant therapy $\geqslant 5$ days before the

\section{AFFLLIATIONS}

Depts of *Medical Imaging

"Internal Medicine,

"Laboratory Medicine,

+Pathology, and

${ }^{f}$ Medical Research, National Taiwan University Hospital and National

Taiwan University College of

Medicine, and

${ }^{5}$ National Center of Excellence for

General Clinical Trial and Research,

National Taiwan University Hospital and College of Public Health,

National Taiwan University, Taipei,

Taiwan.

CORRESPONDENCE

P-C. Yang

Dept of Internal Medicine

National Taiwan University Hospital and National Taiwan University

College of Medicine

7 Chung-Shan South Road

Taipei 100

Taiwan

Fax: 886223582867

E-mail: pcyang@ntu.edu.tw

Received:

April 012007

Accepted after revision:

September 142007

STATEMENT OF INTEREST

None declared. 
CT-guided biopsy procedure, and exhibited platelet counts of $>80 \times 10^{3}$ cells $\mu \mathrm{L}^{-1}$ and prothrombin times and activated partial thromboplastin times within the normal range. Blood component therapy was allowed to correct any abnormal coagulation parameters before biopsy. Patients with vascular lesions or lesions of $\leqslant 4 \mathrm{~mm}$ in diameter were excluded. The present study was approved by the hospital institutional review board. Informed consent was obtained from all patients.

\section{Biopsy procedure}

CT-guided TNB using a coaxial needle $[13,16]$ was performed by two thoracic radiologists (Y-C. Chang and W-J. Lee) and senior residents under their supervision. Two CT scanners (HiSpeed and Imatron C-150; GE Healthcare, Milwaukee, WI, USA) were used. After local skin anaesthesia, a 19-gauge coaxial needle (Temno; Bauer Medical, Clearwater, FL, USA) was inserted stepwise under intermittent CT guidance until the margin of the target lesion was reached. A 20-gauge springloaded semiautomatic biopsy needle with a fixed $1.7-\mathrm{cm}$ cutting trough (Temno; Bauer Medical) was placed into the coaxial needle after removal of the stylet. Most biopsy procedures were performed with a single pleural puncture. A maximum of five core specimens were obtained. The obtained tissue cores were inspected visually before making an imprint. For those pieces considered too small, imprints were not made in order to avoid compromising the tissue core for the histopathological diagnosis. Those biopsy procedures resulting in scanty material were excluded because of the potential bias in interpretation of imprint cytology and histopathology. Directional sampling was performed with adjustment of the cutting trough of the biopsy needle within the coaxial needle in order to obtain specimens and avoid vessel puncture.

The biopsy procedure was stopped if blood continuously oozed or haemoptysis occurred during the procedure. Pulmonary haemorrhage or pneumothorax was determined using final limited CT images immediately after TNB. Pulmonary haemorrhage was determined if there was any increased opacity in the needle path or around the lesion compared with CT images taken before insertion of the biopsy needle. Positive precautions were taken with all patients, who, with the puncture site downwards, were observed for $\geqslant 4 \mathrm{~h}$ and followed-up with chest radiograph. Chest tube placement was indicated when the size of the pneumothorax was $>30 \%$ of the vertical length of the erect chest radiograph, and there was clinical evidence of breathing difficulty or desaturation, even after nasal oxygen supply.

\section{Imprint cytology and histopathology}

Imprint smears were made by lightly touching biopsy specimens against slides, which were then air-dried and evaluated using Riu staining $[17,18]$. Each biopsy specimen yielded four to six imprinted slides. There was no on-site cytologist available during the procedure. The slides for imprint cytology were prepared by the radiologist who performed the CT-guided TNB. The slides were air-dried after lightly touching the specimen on the glass slide. The tissue specimens were then placed in formaldehyde solution ( $10 \%$ formalin) for histological examination. The imprint cytology and histopathology were interpreted independently by different cytologists and pathologists. In those patients who were suspected of having pulmonary infection from clinical and imaging findings, additional tissue culture was performed. For cytological interpretation, Riu staining was performed by cytologists (S-H. Kuo and I-S. Jan), who were responsible for its interpretation. All imprint cytology was carried out using light microscopy and no immunocytochemistry was performed. Histopathological specimens were evaluated with haematoxylin-eosin staining using light microscopy by on-duty pathologists, who neither interpreted nor knew the results of imprint cytology.

A definite TNB diagnosis was defined as a confirmed histopathological diagnosis from surgical resection, microbiology or clinical course after follow-up of $\geqslant 3$ yrs. Definite TNB diagnoses of lung cancer from patients unfit for surgery were based on positive cytology or histopathology. Patients with a clinical diagnosis of infection and nonspecific benign histopathological findings on CT-guided TNB were excluded from the analysis if there was no microbiological evidence of diagnosis, even if there was no change or disappearance of the lesions after 2 yrs. Patients with nonspecific benign histopathological findings from CT-guided TNB who later received a surgical diagnosis were included in the analysis. The interpretation of the imprint cytology results was divided into four categories: 1) positive for malignant cells; 2) suspicious for malignancy; 3) negative for malignant cells; and 4) nondiagnostic due to insufficient cellularity. As previously reported, in the analysis, category 2 was considered positive for malignancy [13]. Patients with category 4 imprint cytology results and those with nondiagnostic histopathology from core biopsy were excluded from analysis. All imprint cytology and histopathology results were reviewed independently by a cytologist (I-S. Jan) and a pathologist (C-H. Hsiao).

\section{Statistical analysis}

The diagnostic accuracy, sensitivity, specificity, positive predictive value (PPV) and negative predictive value (NPV) of TNB using imprint cytology plus histology were compared with those for histology alone and imprint cytology alone. The diagnostic accuracy of TNB was also evaluated in small $(\leqslant 1.5 \mathrm{~cm})$, medium $(1.5-3.0 \mathrm{~cm})$ and large $(>3.0 \mathrm{~cm})$ lesions. McNemar's test was used to compare the diagnostic accuracy of the different methods [19]. Stepwise logistic regression analysis was used to analyse the factors associated with the diagnostic accuracy of TNB [20]. A two-tailed p-value of $\leqslant 0.05$ was considered significant.

\section{Comparison with ultrasound-guided lung biopsy}

Since US was widely used before the advent of CT-guided lung biopsy in the National Taiwan University Hospital, analysis of the results of US-guided biopsy performed before CT-guided TNB was performed for the patient group.

\section{RESULTS}

\section{Patient characteristics}

Between October 1997 and June 2004, 582 out of 622 consecutive patients who underwent CT-guided coaxial core needle biopsy with imprint cytology plus histopathology received a definite diagnosis of their thoracic lesions. Patient demographics and the characteristics of their thoracic lesions are shown in table 1 . The mean age was 62.8 yrs. The mean diameter of the thoracic lesions was $3.6 \mathrm{~cm}$ (range 


\begin{tabular}{lc} 
TABLE 1 Demographics and lesion characteristics \\
Subjects $\mathbf{n}$ & 582 \\
Age yrs & $62.8 \pm 13.5(18.0-91.0)$ \\
Lesion size cm & $3.6 \pm 2.0(0.5-13.0)$ \\
Sex & \\
Male & $335(57.6)$ \\
Female & $247(42.4)$ \\
Lesion pathology & \\
Benign & $151(25.9)$ \\
Malignant & $431(74.1)$ \\
Lesion location & \\
Right upper lobe & $171(29.38)$ \\
Left upper lobe & $126(21.65)$ \\
Right lower lobe & $124(21.31)$ \\
Left lower lobe & $80(13.75)$ \\
Right middle lobe & $58(9.97)$ \\
Anterior mediastinum & $12(2.06)$ \\
Right middle and lower lobes & $4(0.69)$ \\
Middle mediastinum & $3(0.52)$ \\
Posterior mediastinum & $3(0.52)$ \\
Left upper and lower lobes & $1(0.17)$ \\
\hline
\end{tabular}

Data are presented as mean \pm SD (range) or $n(\%)$, unless otherwise stated.

$0.5-13.0 \mathrm{~cm})$. There were 65 small $(\leqslant 1.5 \mathrm{~cm}), 243$ medium $(1.5-3.0 \mathrm{~cm})$, and 274 large $(>3.0 \mathrm{~cm})$ lesions. The diagnoses of 582 thoracic lesions are shown in table 2, including 431 (74.1\%) malignant lesions and 151 (25.9\%) benign lesions.

\section{Diagnostic accuracy, sensitivity and specificity}

The overall diagnostic accuracy, sensitivity, specificity, PPV and NPV of TNB using combined imprint cytology and histopathology compared with histopathology or imprint cytology alone are shown in table 3. Combined imprint cytology and histopathology improved the diagnostic accuracy of CT-guided TNB to $96.4 \%$ (sensitivity $96.5 \%$; specificity $96.0 \% ; \mathrm{p}<0.05$ ) compared with imprint cytology alone (diagnostic accuracy $92.3 \%$; sensitivity $91.0 \%$; specificity $96.0 \%$ ) or histopathology alone (diagnostic accuracy 93.0\%; sensitivity $90.5 \%$; specificity $100 \%$; table 3). Subgroup analysis indicated that this improvement was significant in lesions of $\leqslant 3 \mathrm{~cm}$ in diameter (table 3). Multivariate analyses showed that various factors associated with decreased diagnostic accuracy of TNB were no longer significant after adding imprint cytology to histopathology in CT-guided TNB (table 4).

Among the 582 patients, false-negatives for malignancy occurred in $34(5.8 \%)$ for imprint cytology (mean $\pm \mathrm{SD}$ (range) size $3.8 \pm 2.3 \mathrm{~cm}(0.5-9 \mathrm{~cm})$ ) and $38(6.5 \%)$ for core histopathology (size $4.2 \pm 2.6 \mathrm{~cm}(0.5-12 \mathrm{~cm})$ ). The reasons for falsenegative cytology included unknown cause $(n=10)$, scanty cellularity $(n=9)$, needle position $(n=7)$, surrounding or superimposed inflammation $(n=4)$, severe necrosis $(n=2)$, mucinous nature of tumour $(\mathrm{n}=1)$ and coexisting tuberculosis with lung cancer $(n=1)$. Similar reasons for false-negative core histopathology were found, including needle position $(n=17)$, small specimen $(n=8)$, surrounding inflammation $(n=5)$, tumour necrosis $(n=3)$, pre-existing pneumothorax $(n=2)$,

\section{TABLE 2 Diagnosis of intrathoracic lesions}

$\begin{array}{lc}\text { Subjects } & 58 \\ \text { Malignant } & 43 \\ \text { Adenocarcinoma } & 277 \\ \text { Squamous cell carcinoma } & 40 \\ \text { Small cell carcinoma } & 21 \\ \text { Poorly differentiated carcinoma } & 10 \\ \text { Unclassified nonsmall cell lung cancer } & 20 \\ \text { Metastasis } & 41 \\ \text { Lymphoma } & 7 \\ \text { Other malignant } & 15 \\ \text { Benign } & 15 \\ \text { Pulmonary tuberculosis and NTM infection } & 69 \\ \text { Pneumonia } & 21 \\ \text { Cryptococcus infection } & 12 \\ \text { Fungal infection other than cryptococcus } & 6 \\ \text { Lung abscess } & 6 \\ \text { Benign thymic tumour } & 4 \\ \text { Hamartoma } & 4 \\ \text { Pneumoconiosis } & 3 \\ \text { Sclerosing haemangioma } & 3 \\ \text { Benign neurogenic tumour } & 3 \\ \text { Nonspecific benign } & 3 \\ \text { Other benign } & \end{array}$

Data are presented as n. NTM: nontuberculous mycobacteria. *: includes malignant thymic tumour $(n=4)$, adenosquamous carcinoma $(n=2)$, large cell carcinoma $(n=2)$, adenoid cystic carcinoma $(n=1)$, carcinocarcinoma $(n=1)$, leiomyosarcoma $(n=1)$, carcinoid $(n=1)$, sarcoma $(n=1)$, mesothelioma $(n=1)$ and germ cell tumour $(n=1) ; \bullet$ : the definite diagnoses were obtained from surgical specimens, and included organising pneumonia $(n=11)$, anthrocosis $(n=2)$ and fibrotic nodule $(n=2) ;^{+}:$includes teratoma $(n=1)$, fibromatosis $(n=1)$, inflammatory pseudotumour $(n=1)$, old parasite infection $(n=1)$ and sarcoidosis $(n=1)$

coexisting tuberculosis with lung cancer $(\mathrm{n}=2)$ and mucinous nature of tumour $(n=1)$. False positives for malignancy occurred in six $(1.0 \%)$ imprint cytology results $(4.55 \pm 3.13 \mathrm{~cm}$ $(1-9 \mathrm{~cm})$ ), including three patients with pulmonary tuberculosis and three with pneumonia. False-positives did not occur for histopathology. All of the six false-positive results of imprint cytology were interpreted as suspicious for malignancy because of only a small cluster of suspicious cells and scanty cellularity $(n=2)$ and the presence of chronic inflammation $(n=4)$. Of the 582 lung lesions studied, 11 were reported as suspicious for malignancy by imprint cytology. Five out of the 11 patients with suspicious imprint cytology were correctly diagnosed, including three with lung cancer, one with thymic carcinoma and one with metastatic lung cancer.

Of the patients, 27 (21 false-negative and six false-positive; $4.6 \%$; fig. 1a) received incorrect diagnoses from imprint cytology (fig. 1b) but correct diagnoses from histopathology (fig. 1c). Another $25(4.3 \%)$ patients (fig. 2a) received correct diagnoses from imprint cytology (fig. 2b) but incorrect diagnoses from core histopathology (fig. 2c). Eleven (1.9\%) patients (size $4.11 \pm 2.83 \mathrm{~cm}(0.5-9 \mathrm{~cm})$ ) received incorrect diagnoses from both imprint cytology and core histopathology, including nine lung cancers and two metastases. 


\begin{tabular}{|c|c|c|c|c|c|c|}
\hline Lesion size $\mathrm{cm}$ & Subjects & Sensitivity \% & Specificity \% & PPV \% & NPV \% & Accuracy \% \\
\hline$\leqslant 1.5$ & $65(11.2)$ & & & & & \\
\hline Imprint cytology & & 82.4 & 100.0 & 100.0 & 83.8 & 90.8 \\
\hline Histopathology & & 82.4 & 100.0 & 100.0 & 83.8 & 90.8 \\
\hline $1.5-3.0$ & $243(41.7)$ & & & & & \\
\hline Imprint cytology & & 93.8 & 98.5 & 99.4 & $85 . .5$ & 95.1 \\
\hline Histopathology & & 91.0 & 100.0 & 100.0 & $80 . .5$ & 93.4 \\
\hline Both & & 97.2 & 98.5 & 99.4 & 92.9 & $97.5^{\star}$ \\
\hline$>3.0$ & $274(47.1)$ & & & & & \\
\hline Imprint cytology & & 90.0 & 90.7 & 97.5 & 69.0 & 90.1 \\
\hline Histopathology & & 90.5 & 100.0 & 100.0 & 78.7 & 93.0 \\
\hline Both & & 96.5 & 96.0 & 98.6 & 90.6 & $96.4^{\star}$ \\
\hline
\end{tabular}

Data are presented as $n$ (\%), unless otherwise stated. PPV: positive predictive value; NPV: negative predictive value. *: $p<0.05$ versus imprint cytology/histopathology (McNemar's test; except for both versus histopathology in the lesion size $>3.0 \mathrm{~cm}$ group $(p=0.1336)$ ). $p>0.05$ for all comparisons between imprint cytology and histopathology (McNemar's test).

Of the 582 patients, 43 underwent US-guided lung biopsy before CT-guided TNB. Six $(14.0 \%)$ of them failed to receive a correct diagnosis from US-guided biopsy due to the lack of an appropriate echo window but obtained correct diagnoses on both imprint cytology and histopathology using CT-guided TNB. Of the remaining 37 patients, 13 (30.2\%) obtained correct diagnoses from US-guided biopsy. In contrast, correct

\begin{tabular}{lll} 
TABLE $4 \begin{array}{l}\text { Multiple logistic regression analyses of factors } \\
\text { associated with the diagnostic accuracy of } \\
\text { computed tomography-guided small-bore } \\
\text { coaxial core needle thoracic biopsy using imprint } \\
\text { cytology, histopathology or both\# }\end{array}$ & \\
& OR (95\% Cl) & p-value \\
\hline & & \\
\hline & & \\
Imprint cytology & $0.445(0.157-1.261)$ & 0.1277 \\
Lesion size $1.5-3.0 \mathrm{~cm}$ versus $\leqslant 1.5 \mathrm{~cm}$ & $0.852(0.327-2.219)$ & 0.7435 \\
Lesion size $>3.0 \mathrm{~cm}$ versus $\leqslant 1.5 \mathrm{~cm}$ & $0.785(0.633-0.973)$ & 0.0272 \\
Needle length in lung & $0.372(0.150-0.918)$ & 0.0319 \\
Benign versus malignant lesion & & \\
Histopathology & $0.468(0.157-1.586)$ & 0.2392 \\
Lesion size $1.5-3.0 \mathrm{~cm}$ versus $\leqslant 1.5 \mathrm{~cm}$ & $0.447(0.155-1.485)$ & 0.1986 \\
Lesion size $>3.0 \mathrm{~cm}$ versus $\leqslant 1.5 \mathrm{~cm}$ & $0.954(0.772-1.165)$ & 0.6668 \\
Needle length in lung & $0.039(0.000-0.220)$ & $<0.0001$ \\
Benign versus malignant lesion & & \\
Both & & \\
Lesion size $1.5-3.0 \mathrm{~cm}$ versus $\leqslant 1.5 \mathrm{~cm}$ & $0.856(0.166-4.411)$ & 0.8529 \\
Lesion size $>3.0 \mathrm{~cm}$ versus $\leqslant 1.5 \mathrm{~cm}$ & $1.603(0.342-7.505)$ & 0.5493 \\
Needle length in lung & $0.749(0.545-1.030)$ & 0.0750 \\
Benign versus malignant lesion & $1.111(0.410-3.009)$ & 0.8354 \\
\hline
\end{tabular}

OR: odds ratio; $\mathrm{Cl}$ : confidence interval. ${ }^{*}: \mathrm{n}=582$. diagnosis from both imprint cytology and histopathology was obtained in $35(81.4 \%)$ out of 43 patients undergoing CTguided TNB. Correct diagnosis using CT-guided TNB for malignancy was increased to $88.4 \%$ if either imprint cytology or histopathology results were positive. Of the 13 patients with a correct diagnosis from US-guided biopsy, false-negative results were found in one with histopathology alone and in one with combined histopathology and imprint cytology. Of the 24 patients with false-negative US-guided biopsy results, four gave false-negative results with combined imprint cytology and histopathology and two gave false negative results with imprint cytology alone but a correct diagnosis from histopathology with CT-guided TNB.

\section{Complications of CT-guided thoracic biopsy}

Only eight $(1.4 \%)$ out of 582 patients had clinically significant complications requiring treatment after CT-guided TNB. Of these, six $(1.0 \%)$ required thoracic tube placement for haemothorax (three patients) or tension pneumothorax (three patients). One patient underwent thoracotomy for a haemopneumothorax occurring immediately after TNB due to progressive hypotension. One patient died of pulmonary embolism after successful CT-guided TNB because of discontinuation of anticoagulant therapy for chronic pulmonary thromboembolism for 5 days for CT-guided biopsy.

CT evidence of pulmonary haemorrhage was found in 273 $(46.9 \%)$ patients; 214 of these had malignant lesions and 59 benign lesions $(p=0.025)$. Haemoptysis occurred in $124(21.3 \%)$ patients without haemothorax. Three patients showed haemothorax but no haemoptysis. Pneumothorax occurred in 221 $(38.0 \%)$ patients. There was no significant difference in the incidence rates of pneumothorax (38.1 versus $37.8 \%$; $p=0.9474$ ) or haemoptysis (21.4 versus $21.2 \%$; $=0.9683$ ) in patients with 

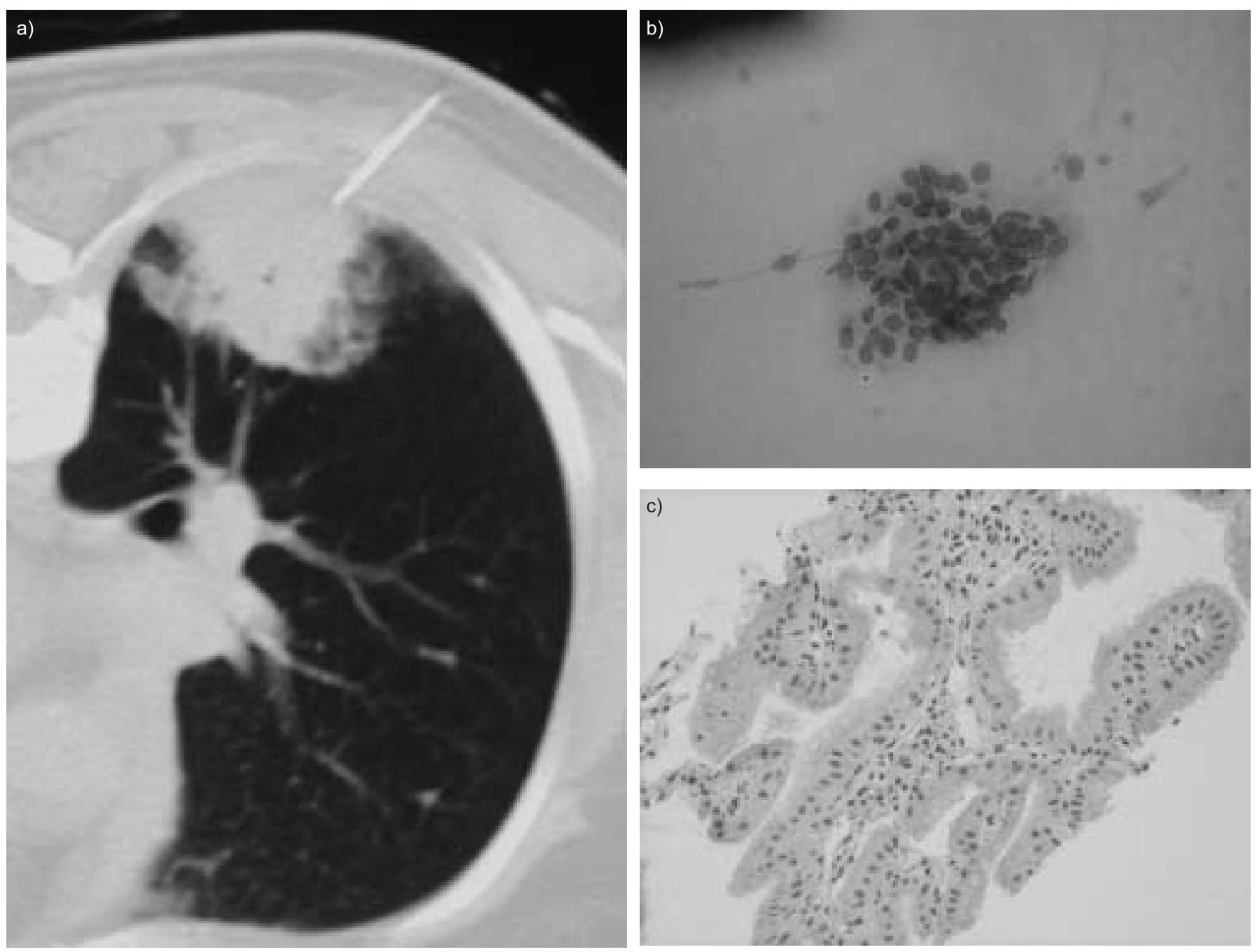

FIGURE 1. Lung cancer in a 54-yr-old female. Example of a false-negative result from imprint cytology but true-positive result from histopathology. a) Axial nonenhanced prone computed tomography showing guiding needle embedded in the periphery of a mass in the right lower lobe. b) Photomicrograph showing no malignant cells on imprint cytology (Riu stain). c) Photomicrograph showing a bronchioloalveolar carcinoma composed of tall and mucinous glandular tumour cells without ciliation (haematoxylin-eosin stain).

malignant versus benign lesions. The incidence rates of pulmonary haemorrhage were $60 \%$ (39 out of 65 ) in small lesions $(\leqslant 1.5 \mathrm{~cm})$ versus $45.3 \%$ (234 out of 517$)$ in larger lesions $(>1.5 \mathrm{~cm} ; \mathrm{p}=0.0248)$.

\section{DISCUSSION}

Percutaneous TNB is relatively safe and accurate for the diagnosis of pulmonary and mediastinal lesions [1-5]. The diagnostic accuracy of TNB for pulmonary nodules ranges 76$95 \%$ [1-8], and decreases in smaller lesions $(<1.5-2 \mathrm{~cm})[2,7,8$, 21]. Methods reported to improve the diagnostic accuracy of image-guided TNB include frozen-section diagnosis [9], FNA [10] and CT fluoroscopy [22, 23]. Frozen-section diagnosis with CT-guided TNB is time-consuming, and improvement of the diagnostic accuracy may be limited [9]. Combined FNA and core biopsy may raise the diagnostic yield to $97.1 \%$ and the precise diagnosis to $94.2 \%$ using CT fluoroscopy [10]. Realtime CT fluoroscopy may be one of the best adjuncts to TNB. It can monitor in real-time the entry of the needle into lesions with excellent diagnostic sensitivity (95.1\%) and accuracy (96.2\%) using vacuum-assisted devices [24]. However, special equipment is required and the radiologists' radiation exposure may be increased [25].

To the best of the present authors' knowledge, this is the first report of imprint cytology and CT-guided coaxial core needle biopsy of thoracic lesions in a large group of patients. It was found that combined imprint cytology and histology improved the diagnostic accuracy of CT-guided TNB to $96.4 \%$ (sensitivity $96.5 \%$; specificity $96.0 \%$; $\mathrm{p}<0.05$ ) compared with imprint cytology alone (diagnostic accuracy $92.3 \%$; sensitivity $91.0 \%$; specificity $96.0 \%$ ) or histopathology alone (diagnostic accuracy $93.0 \%$; sensitivity $90.5 \%$; specificity $100 \%$ ) in 582 patients.

In the present study, the diagnostic accuracy using imprint cytology and CT-guided coaxial core TNB was comparable to that obtained using CT fluoroscopy-guided FNA and core biopsy with (sensitivity 95.1\%; specificity 100\%; diagnostic accuracy $96.2 \%$ ) or without (sensitivity $94 \%$; specificity $100 \%$; 

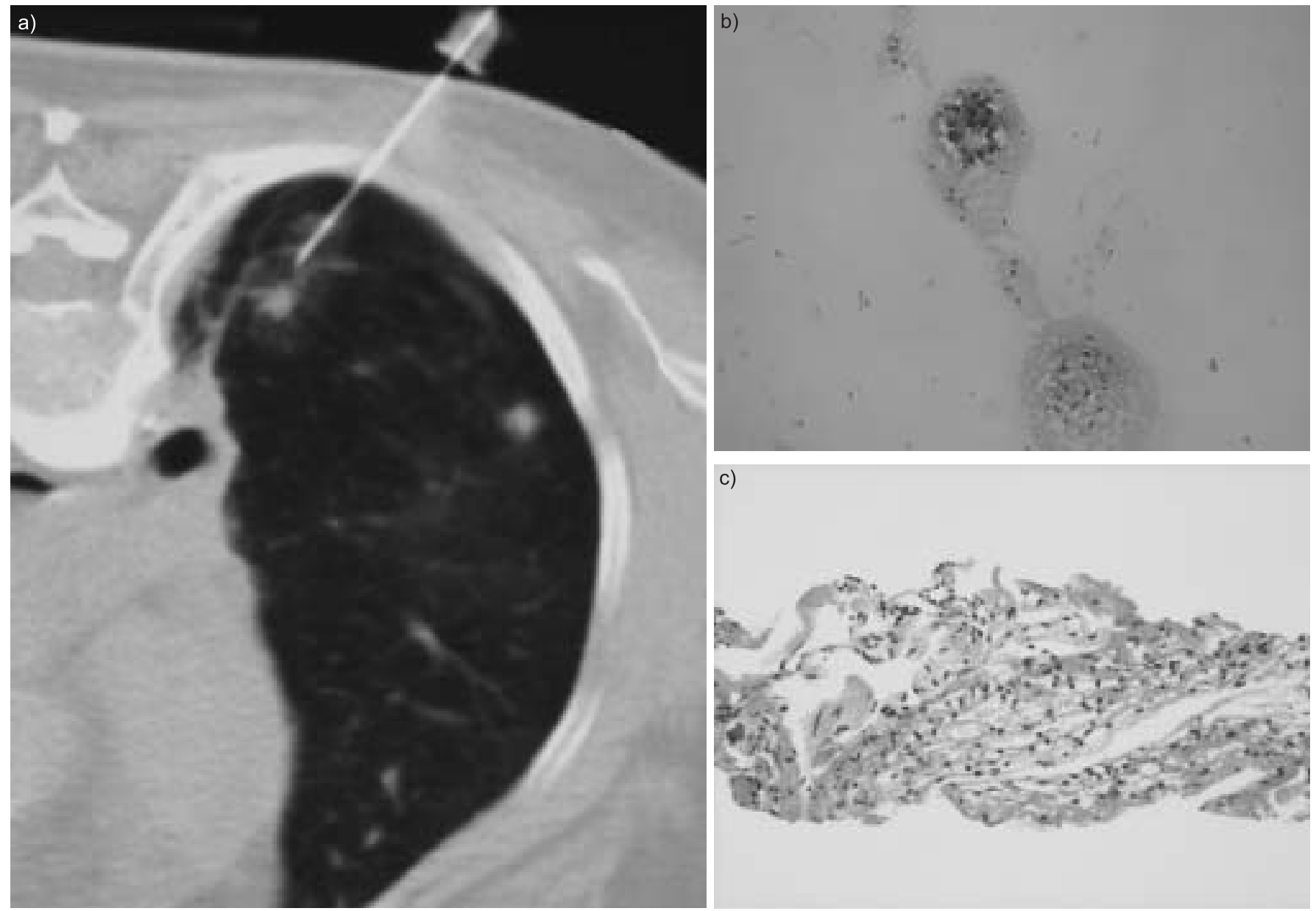

FIGURE 2. Lung cancer in a 59-yr-old female. Example of a false-negative result from histopathology but true-positive result from imprint cytology. a) Axial nonenhanced prone computed tomography showing the placement of a guiding needle for biopsy of one of the small lesions in the right lower lobe. b) Photomicrograph showing a cluster of cells consistent with adenocarcinoma (Riu stain). c) Photomicrograph showing bronchopneumonia with focal fibrin deposition and neutrophilic infiltration in the alveolar spaces and no malignant cells (haematoxylin-eosin stain).

diagnostic accuracy 96.2\%) vacuum assistance [22, 24]. However, CT-guided TNB can avoid exposure of the radiologist to extra radiation compared with CT fluoroscopyguided TNB. Imprint cytology and CT-guided TNB also gave a higher diagnostic accuracy (88.4 versus $30.2 \%$ ) than US-guided biopsy in the present patients.

The reasons for false-negative results from either imprint cytology or histopathology with CT-guided TNB were similar, including needle position, surrounding inflammation and tumour necrosis. The main causes of false-negative cytology were uncertain, but scanty cellularity, surrounding or superimposed inflammation, and character of tumour, such as necrosis or mucin production, may be important. Small specimens, existing pneumothorax during the procedure, severe tumour necrosis or a mucin-producing tumour may cause false-negative histopathology. Appropriate planning of the needle tract and the target for biopsy are important in order to avoid false-negative results.

In the present study, $4.3 \%$ (25 out of 582) of all patients received correct diagnoses from imprint cytology but not from histopathology. Imprint cytology is considered an excellent method for giving correct and rapid diagnosis without compromising the tissue specimen for histopathology [13, 26]. Using core roll preparations from the core biopsy plus the corresponding FNA smears, the diagnostic yield of neoplastic lung lesions was better than with FNA alone, due to better cellularity and morphology [26]. HAYASHI et al. [9] also demonstrated that cytology can yield the correct diagnosis from small lesions with CT-guided biopsy. МотOMURA et al. [11] reported that imprint cytology can detect micrometastasis more precisely than final paraffin sections evaluated by haematoxylin and eosin staining in breast cancer. Therefore, touch imprint cytology could be superior to conventional histopathology in the identification of a small proportion of cancer cells against a background of nonmalignancy [11, 12]. Imprint cytology gave a $1 \%$ false-positive rate due to suspicious cytology in the present study, comparable to the results of prior reports [27, 28].

Multivariate analysis in the present study showed that factors that decreased the diagnostic accuracy of imprint cytology or histopathology following CT-guided TNB were no longer 
significant after combining both cytology and histopathology. The diagnostic accuracy of TNB decreases with small lesion size, as has been reported in several studies [2, 6-8, 21]. The significant decreases in the diagnostic accuracy of CT-guided TNB with FNA or single-shot cutting biopsy to $67-74 \%$ in pulmonary lesions of $<2.0 \mathrm{~cm}[2,7,21]$ may be associated with sampling errors of small lesions [21]. Coaxial multiple-shot cutting biopsy increases the diagnostic accuracy to $84 \%$ in pulmonary lesions of $<1.5 \mathrm{~cm}$ [8]. However, the diagnostic accuracy of small thoracic lesions was excellent with $(96.9 \%)$ or without $(90.8 \%)$ adding imprint cytology to CT-guided core biopsy in the present study.

In the present study, a potential limitation was the inability to demonstrate that imprint cytology plus histopathology, compared with histopathology alone, can significantly improve the diagnostic accuracy of CT-guided TNB in large thoracic lesions $(>3.0 \mathrm{~cm})$. However, the diagnostic accuracy was significantly improved in small and medium-sized lesions, and this trend was also obvious in large lesions. This may be due to less compensatory effect of imprint cytology for histopathology in large thoracic lesions. This discrepancy might be due to the fact that the diagnostic accuracy of imprint cytology was relatively lower in large lesions $(>3.0 \mathrm{~cm})$ compared with medium-sized lesions. Whether imprint cytological diagnosis should be performed during TNB in order to reduce the number of biopsy procedures and lower the rate of procedural complications may require further investigation.

The pneumothorax rate of $38.0 \%$ after TNB in the present study is similar to the $21-37 \%$ reported in other studies $[2,5,9$, $10,21,22,24]$, with a range of $6.8-54 \%[1,8]$. The rate of pneumothorax after TNB requiring chest tube treatment $(1 \%)$ in the present study is consistent with the $2.9-12 \%$ reported [4, $7,10,21-23]$, with a range of $0-15 \%[1,6]$. The present incidence of pulmonary parenchymal haemorrhage of $46.9 \%$ after TNB is slightly higher than in previous reports (5.1-42\%) $[1,2,10]$, whereas the haemoptyis incidence of $21.3 \%$ was much higher than the $2.2-6.4 \%$ in other reports [2, 7-10, 24]. Most of the bleeding complications were self-limiting and appeared as asymptomatic ground-glass attenuation on CT. Patients with smaller lesions and malignant lesions showed significantly higher incidence rates of pulmonary haemorrhage in the present study. It was assumed that patient selection might be one of the factors causing more bleeding complications since lesions touching pleura, which may have significantly less chance of bleeding [29], were assigned for USguided biopsy in the National Taiwan University Hospital. Whether imprint cytological diagnosis should be performed during TNB in order to reduce the number of biopsy procedures and lower the rate of procedural complications may require further investigations.

In conclusion, imprint cytology can improve the diagnostic accuracy of computed tomography-guided transthoracic coaxial core biopsy.

\section{ACKNOWLEDGEMENTS}

The authors would like to thank L-C. Wu for assistance with statistical computing.

\section{REFERENCES}

1 Klein JS, Salomon G, Stewart EA. Transthoracic needle biopsy with a coaxially placed 20-gauge automated cutting needle: results in 122 patients. Radiology 1996; 198: 715-720.

2 Tsukada H, Satou T, Iwashima A, Souma T. Diagnostic accuracy of CT-guided automated needle biopsy of lung nodules. AJR Am J Roentgenol 2000; 175: 239-243.

3 García Río F, Díaz Lobato S, Pino JM, et al. Value of CTguided fine needle aspiration in solitary pulmonary nodules with negative fiberoptic bronchoscopy. Acta Radiol 1994; 35: 478-480.

4 Sider L, Davis TM. Hilar masses: evaluation with CTguided biopsy after negative bronchoscopic examination. Radiology 1987; 164: 107-109.

5 Swischuk JL, Castaneda F, Patel JC, et al. Percutaneous transthoracic needle biopsy of the lung: review of 612 lesions. J Vasc Interv Radiol 1998; 9: 347-352.

6 Haramati LB. CT-guided automated needle biopsy of the chest. AJR Am J Roentgenol 1995; 165: 53-55.

7 Laurent F, Latrabe V, Vergier B, Montaudon M, Vernejoux JM, Dubrez J. CT-guided transthoracic needle biopsy of pulmonary nodules smaller than $20 \mathrm{~mm}$ : results with an automated 20-gauge coaxial cutting needle. Clin Radiol 2000; 55: 281-287.

8 Yeow KM, Tsay PK, Cheung YC, Lui KW, Pan KT, Chou AS. Factors affecting diagnostic accuracy of CT-guided coaxial cutting needle lung biopsy: retrospective analysis of 631 procedures. J Vasc Interv Radiol 2003; 14: 581-588.

9 Hayashi N, Sakai T, Kitagawa M, et al. CT-guided biopsy of pulmonary nodules less than $3 \mathrm{~cm}$ : usefulness of the springoperated core biopsy needle and frozen-section pathologic diagnosis. AJR Am J Roentgenol 1998; 170: 329-331.

10 Yamagami T, Iida S, Kato T, Tanaka O, Nishimura $T$. Combining fine-needle aspiration and core biopsy under CT fluoroscopy guidance: a better way to treat patients with lung nodules? AJR Am J Roentgenol 2003; 180: 811-815.

11 Motomura $\mathrm{K}$, Inaji $\mathrm{H}$, Komoike $\mathrm{Y}$, et al. Intraoperative sentinel lymph node examination by imprint cytology and frozen sectioning during breast surgery. Br J Surg 2000; 87: 597-601.

12 Okubo K, Kato T, Hara A, Yoshimi N, Takeda K, Iwao F. Imprint cytology for detecting metastasis of lung cancer in mediastinal lymph nodes. Ann Thorac Surg 2004; 78: 1190-1193.

13 Paulose RR, Shee CD, Abdelhadi IA, Khan MK. Accuracy of touch imprint cytology in diagnosing lung cancer. Cytopathology 2004; 15: 109-112.

14 Liao WY, Jerng JS, Chen KY, Chang YL, Yang PC, Kuo SH. Value of imprint cytology for ultrasound-guided transthoracic core biopsy. Eur Respir J 2004; 24: 905-909.

15 Yang PC. Ultrasound-guided transthoracic biopsy of the chest. Radiol Clin North Am 2000; 38: 323-343.

16 Chang YC, Wang HC, Yang PC. Usefulness of computed tomography-guided transthoracic small-bore core biopsy in the presence of a pneumothorax. J Thorac Imag 2003; 18: 21-26.

17 Riu $\mathrm{CH}$. On the studies of the methods of staining blood film. J Nigada Med Assoc 1956; 70: 635-643.

18 Lee CH, Liu CY, Wang CH, Ho YS, Chen TC. Use of Riu stain in the immediate interpretation of bronchial brushing cytology. Comparison with Papanicolaou stain and histopathology. Acta Cytol 1997; 41: 1171-1177. 
19 Rosner B. Two-sample test for binomial proportions for matched-pair data (McNemar's test). In: Rosner B, ed. Fundamentals of Biostatistics. 5th Edn. Pacific Grove, Duxbury, 2000; pp. 376-384.

20 Hosmer DW, Lemeshow S. Applied Logistic Regression. 2nd Edn. Hoboken, John Wiley \& Sons, 2000.

$21 \mathrm{Li} \mathrm{H}$, Boiselle PM, Shepard JO, Trotman-Dickenson B, McLoud TC. Diagnostic accuracy and safety of CT-guided percutaneous needle aspiration biopsy of the lung: comparison of small and large pulmonary nodules. AJR Am J Roentgenol 1996; 167: 105-109.

22 Muehlstaedt M, Bruening R, Diebold J, Mueller A, Helmberger T, Reiser M. CT-fluoroscopy-guided transthoracic needle biopsy: sensitivity and complication rate in 98 procedures. J Comput Assist Tomogr 2002; 26: 191-196.

23 Hirose T, Mori K, Machida S, Tominaga K, Yokoi K, Adachi M. Computed tomographic fluoroscopy-guided transthoracic needle biopsy for diagnosis of pulmonary nodules. Jpn J Clin Oncol 2000; 30: 259-262.

24 Yamagami $\mathrm{T}$, Iida $\mathrm{S}$, Kato $\mathrm{T}$, et al. Usefulness of new automated cutting needle for tissue-core biopsy of lung nodules under CT fluoroscopic guidance. Chest 2003; 124 147-154.

25 Paulson EK, Sheafor DH, Enterline DS, McAdams HD, Yoshizumi TT. CT fluoroscopy-guided interventional procedures: techniques and radiation dose to radiologist. Radiology 2001; 220: 161-167.

26 Chandan VS, Zimmerman K, Baker P, Scalzetti E, Khurana KK. Usefulness of core roll preparations in immediate assessment of neoplastic lung lesions: comparison to conventional CT scan-guided fine needle aspiration cytology. Chest 2004; 126: 739-743.

27 Manhire A, Charig M, Clelland C, et al. Guidelines for radiologically guided lung biopsy. Thorax 2003; 58: 920-936.

28 Denley H, Singh N, Clelland CA. Transthoracic fine needle aspiration cytology of lung for suspected malignancy: an audit of cytological findings with histopathological correlation. Cytopathology 1997; 8: 223-229.

29 Yeow KM, Su IH, Pan KT, et al. Risk factors of pneumothorax and bleeding: multivariate analysis of 660 CT-guided coaxial needle lung biopsies. Chest 2004; 126: 748-754. 